\title{
Effect of Salicylic Acid, Calcium Chloride and Calcium Lactate Applications on Quality Attributes of Minimally-Processed 'Wonderful' Pomegranate Arils
}

\author{
Samar A.M.A. SHAARAWI ${ }^{1 *}$, Assem S.E. SALEM ${ }^{2}$, \\ Ibrahim M.K. ELMAGHRABY², Eman A.A. ABD EL-MONIEM ${ }^{3}$ \\ ${ }^{1}$ Horticulture Research Institute, Agricultural Research Center, Fruit HandlingResearch Department, Giza, Egypt; sshaarawi@hotmail.com (*correspondingauthor) \\ ${ }^{2}$ Agricultural Research Center, Central Laboratory of Organic_Agriculture,Giza, Egypt; assemsalama@hotmail.com; Ibrabim_elmaghraby@yahoo.com \\ ${ }^{3}$ National Research Centre, Horticulture Technology_Department,Egypt; eman_abdel_moniem@yahoo.com
}

\begin{abstract}
'Wonderful' pomegranate arils were treated with $0.5 \%$ and $1 \%$ of calcium chloride, $0.5 \%$ and $1 \%$ of calcium lactate, and salicylic acid ( 1 and $2 \mathrm{mM}$ ), then treated and untreated (control) arils packaged in clean (sterilized) plastic containers. Fresh produce was then stored at $5 \pm 1^{\circ} \mathrm{C}$. Assessment of arils quality was carried out at 3-day intervals by evaluating the following quality parameters: appearance, decay, off odor, flavor, total soluble solids, acidity, anthocyanin and vitamin $\mathrm{C}$ content, firmness, colour development ( $\mathrm{L}^{*}$ value and hue angle), and microbial load. Pomegranate arils treated with salicylic acid, calcium chloride, and calcium lactate maintained a general good quality and appearance up to 12 days of storage at $5 \pm 1{ }^{\circ} \mathrm{C}$, with no visible decay and off odor development. The total microbial population was lower in arils treated with salicylic acid, in comparison to treatments with calcium chloride, calcium lactate and control arils. All treatments scored above the limit of marketability, maintaining good quality of fresh-cut produce during storage. However, the use of salicylic acid and calcium chloride helped to keep a better overall quality of arils at the end of the 12 -day storage at $5 \pm 1{ }^{\circ} \mathrm{C}$.
\end{abstract}

Keywords: calcium chloride, calcium lactate, fresh-cut, pomegranate, Punica granatum L., quality attributes

Abbreviations: Ca-lactate, calcium lactate; SA, salicylic acid; TA, titratable acidity; TSS, total soluble solids

\section{Introduction}

Fresh-cut fruits and vegetables are a relatively new and rapidly developing segment of the fresh produce industry, and the demand is continuously increasing, being the convenience factor and health promoting benefits, associated with their consumption, the main reasons for such an increment (Sanchís et al., 2015). During the last decade, in particular, an interest in the consumption of pomegranates (Punica granatum $\mathrm{L}$.) has been recorded, due to the health benefits produced by the very high content of bioactive phytochemicals of the fruit (Holland et al., 2009; Viuda-Martos et al., 2010). Despite these health benefits, pomegranate consumption is still limited due to the difficulty of extracting the arils, and the inconvenience due to phenolic metabolites which stain the hands during preparation of seeds (Opara et al., 2009). Packaged ready-to-eat arils is a more appealing product to consumers and increases the prospect of both production and consumption (Caleb et al.,2012).
Improperly preparing, packaging and handling fresh-cut fruit may compromise overall quality and decrease consumer acceptability (Beaulieu and Gorny, 2002). Physiological changes may be accompanied by flavor loss, cut surface discoloration, color loss, decay, increased rate of vitamin loss, rapid softening, shrinkage and a shorter storage-life. These attributes reflect visual acceptance and physicochemical properties associated with the product quality. Therefore, proper treatments of the product are essential for the maintenance of such quality (Gorny et al., 2002). Among them, the application of calcium compounds is effective in maintaining the flesh firmness of fresh-cut fruit products. The dipping of fresh-cut products in solutions of 0.5 to $1.0 \%$ calcium chloride is the common method of application (Ponting et al., 1971; Ponting et al., 1972). Calcium is used from long time as an agent for maintaining firmness of whole produce (Poovaiah, 1986), and its subsequent use in fresh-cut production has been an obvious consequence. According to Elyatem and Kader (1984), chilling injury, decay, and weight loss are the most important 
problems, limiting pomegranate storability. Treatments like calcium dipping can help to maintain firmness and visual quality, resulting in a longer shelf life of the fresh-cut products as melon (Aguayo et al., 2008) and apple slices (Aguayo et al., 2010).

Calcium $\left(\mathrm{Ca}^{2+}\right)$ has been extensively reviewed both as an essential element, and for its potential role in maintaining postharvest quality of fruit and vegetable crops by contributing to the linkage between pectic substances within the cell wall (Kirkby and Pilbeaam, 1984; Demarty et al., 1984; Arhtar et al., 2010). The presence of $\mathrm{Ca}^{2+}$ ions increases the cohesion of cell walls (Lara et al., 2004; Kazemi et al., 2011). It is also involved in reducing the rate of senescence and fruit ripening (Ferguson, 1984; White and Broadley, 2003; Mahajan and Dhatt, 2004). A $1 \%$ solution of $\mathrm{CaCl}_{2}$ delayed fruit ripening, improved resistance to fungal attack and maintained structural integrity of cell walls of strawberry during a 10-day storage period at $3^{\circ} \mathrm{C}$ (Lara et al., 2004).

Calcium lactate (Ca-lactate) has recently been shown to be as effective as the chloride form (at higher concentrations) without imparting a bitter flavor (Luna-Guzmán and Barrett, 2000). A $1 \%$ Ca-lactate dip was an effective alternative to ascorbate in fresh-cut 'Bartlett' pears stored 1 to 2 days at $20^{\circ} \mathrm{C}\left(68^{\circ} \mathrm{F}\right)$, while $1 \%$ Ca-lactate plus $2 \%$ ascorbate was the most effective (Gorny et al., 1998). On the other hand, a combination dip with $0.01 \% 4$ hexylresorcinol, $0.5 \%$ ascorbate and $1 \%$ Ca-lactate extended shelf-life of 'Anjou,' 'Bartlett,' and 'Bosc' pear slices for 15 to 30 days (Dong et al., 2000), while kiwifruit slices treated with 2\% Ca-lactate had a shelf-life of 9-12 days (Agar et al., 1999; Massantini and Kader, 1995). In addition, Ca-lactate showed an anti-browning effect, as browning was also reduced in fresh-cut 'Carnival' peaches treated with 1\% Ca-lactate plus 2\% ascorbate (Gorny et al., 1999). Gorny et al. (2002) reported that a postcutting dip ( $\mathrm{pH} 7.0)$ of $2 \%$ ascorbate, $1 \%$ Ca-lactate and $0.5 \%$ $(\mathrm{w} / \mathrm{v})$ cysteine significantly extended shelf-life of 'Bartlett' pear slices by inhibiting loss of firmness and preventing browning.

Salicylic acid (SA) is a natural phenolic compound involved in the regulation of many processes in plant growth and development. Among them, it is noteworthy that SA exhibits a high potential in controlling post-harvest losses of horticultural crops (Asghari and Aghdam, 2010). Moreover, dietary salicylates from fruit and vegetables are described as bioactive compounds with health care potential (Hooper and Cassidy, 2006), and considered as "Generally Recognized As Safe" (GRAS). For its action at minimal concentrations, SA is considered a plant hormone (Raskin, 1992), inhibiting ethylene biosynthesis and delaying fruit senescence (Khademi et al., 2012). Exogenous SA treatment may also induce the expression of pathogenesis-related proteins (Malamy et al., 1990), and establish systemic acquired resistance (Gaffney et al., 1993, Beckers and Spoel, 2006). The application of acetylsalicylic acid (a derivative of SA) slowed down the softening rate of kiwifruit by inhibiting ethylene production and maintaining higher endogenous SA levels (Zhang et al., 2003). SA also prevented the softening of banana and kiwifruit during ripening (Srivastava and Dwivedi, 2000; Zhang et al., 2003). It has been also reported that SA application, either in pre- or post-harvest, reduced fungal decay in sweet cherry (Yao and Tian, 2005; Xu and Tian, 2008), strawberry (Babalar et al., 2007; Shafiee et al., 2010) and peach fruits (Wang et al., 2006). SA can extend the shelf life of the harvested fruit by delaying the development of disease incidence (Terry and Joyce,
2004), through induction of the defense resistance system and stimulation of antioxidant enzymes (Khademi and Ershadi, 2013).

With attention to the risk of improper use of chemicals in postharvest technology and consumer's demand for healthy products, studies on the application of postharvest treatments, along with cold storage, are today considered of strategic importance (Khademi and Ershadi, 2013). In this context, the aim of this study was to determine the effects of calcium chloride $\left(\mathrm{CaCl}_{2}\right), \mathrm{SA}$, and Ca-lactate on qualitative characteristics of minimally processed 'Wonderful' pomegranate arils during cold storage at $5 \pm 1^{\circ} \mathrm{C}$.

\section{Materials and Methods}

\section{Plant material, treatments and evaluations}

The current study was carried out throughout two successive seasons, 2013 and 2014. 'Wonderful' pomegranate (Punica granatum L.) fruits were harvested at maturity stage from a private farm located in the desert road, Giza governorate, Egypt. Fruits were immediately transported to the laboratory, and then were selected in uniformity of weight, size, maturity stage and absence of physical injuries. All preparation steps were applied in clean room conditions. Fruits were washed in sterilized water with $200 \mathrm{ul} \mathrm{l}^{-1}$ of sodium hypochlorite $(\mathrm{NaOCl})$ solution. Fruit husks were processed for aril extraction, the arils were gently removed by hand and then collected and mixed together for uniformity in a clean container. After removing the damaged ones, arils were divided into six portions and subjected to the following treatments in triplicate: (1) for calcium dip treatments, $0.5 \%\left(5 \mathrm{gl}^{-1}\right.$ of $\left.\mathrm{CaCl}_{2}\right)$ and $1 \%\left(10 \mathrm{gl}^{-1}\right.$ of $\left.\mathrm{CaCl}_{2}\right)$ solutions were prepared and arils were dipped in one of the two for $5 \mathrm{~min}$; (2) for Ca-lactate treatments, the same concentrations of $0.5 \%$ and $1 \%$ were applied with 5 min dipping; (3) for SA treatments, arils were dipped in $1 \mathrm{mM}$ or $2 \mathrm{mM}$ solutions for $5 \mathrm{~min}$. Arils dipped in sterile water for $5 \mathrm{~min}$ served as control. After dipping, arils of each treatment were drained in a colander and left for drying using clean cloth absorbent paper. Finally, $110 \mathrm{~g}$ of pomegranate arils from each treatment were packaged in previously sterilized rigid plastic container (PET, diameter: $110 \mathrm{~mm}$, height: $55 \mathrm{~mm}$ ), and then kept in cold storage at $5 \pm 1{ }^{\circ} \mathrm{C}$ and $95 \pm 2 \% \mathrm{RH}$ for up to 12 days. Samples were taken for assessment at day 0 , and then at 3-day intervals during storage. The quality characteristics of arils were evaluated in terms of: (i) total soluble solids (TSS) using digital pocket refractometer (model PAL 1, ATAGO ${ }^{\mathrm{TM}}$, Tokyo Tech.) and expressed as percentage, (ii) titratable acidity (TA) percentage, according to A.O.A.C. (1990), (iii) ascorbic acid content (mg/100 ml), according to Lucas (1944), (iv) total anthocyanin content $(\mathrm{mg} / 100 \mathrm{ml})$ was calorimetrically determined in fruit juice as described by Hsia et al. (1965), (v) texture (firmness) of arils was measured using lefra texture analyzer (Mehteric Stevens, model TA/000), a test speed of 2 $\mathrm{mm}$ and $1 \mathrm{~mm}$ depth. Aril color was determined and expressed as: $\mathrm{L}^{*}$ value, i.e. a measure of lightness, ranging from 0 (black) to +100 (white), and hue angle $\left(\mathrm{h}^{\circ}\right)$, using a Minolta colorimeter CR-40 (Konica Minolta Sensing Inc, Sakai, Japan).

\section{Sensorial overall quality attributes}

Sensory evaluation was performed during storage at 3-day intervals. Panel members were requested to assess fresh quality measurement as follows: 
510

1. Visual quality: based on overall visual appearance, it was evaluated following a 9-point rating scale where 9, excellent; 7, very good; 5, good (limit of consumer acceptability); 3, fair (limit of usability), and 1, extremely poor (Gorny et al., 2002; Medina et al, 2012).

2. Decay: estimated visually using scores, as described by Kader et al. (1973) on 5-1 scale, with reference points of: 5, severe; 4, moderately severe; 3 , moderate; 2 , slight; 1 , none. The score attribution depends on morphological effects such as color change, microorganism effects, smell and decay percentage on arils.

3. Off odors: determined just after opening the package by using a 5-point scale where: 5 , severe; 4 , moderately severe; 3 , moderate; 2, slight; 1 , none. This scale depends on unlike or bad smell (El-Bassiouny, 2003).

4. Flavor: estimated using a 5-point scale where: 5 , fully typical flavor; 4, moderately full; 3 , moderate; 2 , slight; 1 , poor.

\section{Microbialloadstudies}

For media preparation, Nutrient glucose agar (Dowson, 1957) and Rose-bengal agar media (Johnson et al., 1960) were prepared and sterilized by autoclaving at $121^{\circ} \mathrm{C}$ for $20 \mathrm{~min}$, after which the media were allowed to cool to $45-50^{\circ} \mathrm{C}$ before inoculating them with sample material. The serial dilution method was used for the recording of the micro-organism load count. The inoculated plates were placed in two different incubators that had been pre-set at a suitable temperature for both fungi and bacteria.

\section{Microbiological assay}

One gram of pomegranate arils from each treatment were homogenized to obtain the microbial count. Ten-fold serial dilutions were carried out and pour-plate method was used for enumeration of bacteria and fungi from tested samples, since 1.0 $\mathrm{ml}$ of each dilution was pour-plated in triplicate onto appropriate media. Total bacterial counts were enumerated on nutrient glucose agar medium ( 3 g beef extract, 5 g peptone, 8 gglucose, and $20 \mathrm{~g}$ agar per liter) after incubation at $37^{\circ} \mathrm{C}$ for $2448 \mathrm{~h}$. Total molds and yeasts were enumerated on Rose-bengal agar medium (10 g glucose, $5 \mathrm{~g}$ peptone, $1 \mathrm{~g} \mathrm{KH}_{2} \mathrm{PO}_{4}, 0.03 \mathrm{~g}$ Rose-bengal, $0.5 \mathrm{~g}$ $\mathrm{MgSO}_{4}$, and $20 \mathrm{~g}$ agar per liter). Incubation for total mold and yeast counts was performed at $28^{\circ} \mathrm{C}$ for $2-3$ days. The evaluation of the microbial load was performed 3 times during the storage experiment, and results were determined as colony-forming units per gram $\left(\mathrm{CFU} \mathrm{g}^{-1}\right)$ (Sogvar et al., 2016). As for the enumeration of colonies, following incubation, all colonies on dishes containing 30-300 colonies were counted and the results per dilution were calculated. Microbial data were then transformed into logarithms of the number colony forming units $\left(\log \mathrm{CFU} \mathrm{g} \mathrm{g}^{-1}\right)$.

\section{Statistical analysis}

For experiments, complete factorial randomized designs were applied. Treatments were always replicated three times, and the obtained data were statistically analyzed by ANOVA. Mean comparisons were performed by Duncan's Multiple range test at 5\% level (Snedecor and Cochran, 1982).

\section{Results and Discussions}

\section{Totalsolublesolids}

Data reported in Table 1 show that TSS percentages increased, often significantly, by the increasing of the storage period, and this observation was true for both the studied seasons. These results are in accordance with Khademi and Ershadi (2013) who reported that, irrespectively to treatments, soluble sugar content (SSC) of peach fruits increased slightly during storage. Moreover, Arendse et al. (2014) reported that TSS of 'Wonderful' pomegranate fruits increased significantly during storage at the investigated temperature regimes, i.e. after one-month storage TSS increased from 13 to $16.22,15.36,14.84$ and $14.35^{\circ}$ Brix at 5, 7.5, 10 and $21^{\circ} \mathrm{C}$, respectively.

As for the different treatments, arils treated with $1 \% \mathrm{CaCl}_{2}$ in the first season and $2 \mathrm{mM} \mathrm{SA}$ in both seasons showed the highest significant TSS as compared to control. Control arils and 0.5\% $\mathrm{CaCl}_{2}$ treated ones had the lowest values of TSS after 3 days of storage. In the first season, at 12 days of storage, arils treated with $1 \% \mathrm{CaCl}_{2}$ scored the highest TSS percentages as compared to control ones, while, in the second season, $\mathrm{CaCl}_{2}$ at both tested concentrations recorded the highest TSS respecting to the control. Mirdehghan and Ghotbi (2014) indicated that SA applied at 1 $\mathrm{mM}$ significantly increased TSS compared to untreated pomegranate fruits. Results are also in accordance with Srivastava and Dwivedi (2000) who reported that SA treatments increased TSS in banana fruits. They hypothesized that cell walls contain large amounts of polysaccharides, mainly pectin and cellulose, which are digested due to the activity of the cell wall-degrading enzymes, leading to a significant increase in TSS content. In this regard Amith et al. (2015) found that the highest TSS was for pomegranate arils treated with $1 \%$ calcium chloride. On the other side, other reports showed that TSS was not greatly influenced by SA treatment in pomegranate (Sayyari et al., 2009) and mango (Ding et al., 2007). Similarly, Mirdehghan et al. (2012) affirmed that TSS of pomegranate fruit juice was not influenced when fruits were treated with SA, methyl jasmonate, and potassium sulfate.

The effects of SA treatments on the sugar content of fruits and vegetables are controversial. SA treatment had no effect on SSC on fruits of grape (Ranjbaran et al, 2011) and persimmon (Khademi et al., 2012). Asghari and Aghdam (2010) reported that the lower contents of TSS were obtained in kiwifruit treated with $32 \mu \mathrm{ll}^{-1}$ of methyl salicylic acid (MeSA) at the end of cold storage. According with this observation, Mirdehghan and Ghotbi (2014) proposed that MeSA reduces ethylene production, resulting in the decrease of sucrose-phosphate synthase enzyme activity that, in turn, leads to the decrease of sucrose synthesis.

\section{Titratable acidity}

In this study, acidity percentages markedly decreased as storage period increased (Table 2). The reduction in acidity was significant up to 6 days of storage, then continued to decrease but with no significant differences up to 9 days (in the first season), and to the end of storage period (in the second season).

In both the seasons, $\mathrm{CaCl}_{2}$ at $0.5 \%$, Ca-lactate at $0.5 \%$, and $\mathrm{SA}$ at $1 \mathrm{mM}$ showed the lowest acidity percentages in comparison to untreated (control) arils, while $2 \mathrm{mM} \mathrm{SA}$ and $1 \% \mathrm{CaCl}_{2}$ had the highest mean values.

These results are in accordance with those obtained by Khademi and Ershadi (2013) who showed that TA reduced slightly with SA treatments during storage, although the reduction was never significant. Moreover, these results are in agreement with those reported by Sayyari et al. (2009) and Ranjbaran et al. (2011). The observed decrease in acidity could be related to initial response and metabolic activities of the arils during the storage, as suggested by Caleb et al. (2013). On the other hand, other authors reported that TA was not affected by SA on mango 
Table 1. TSS(\%) of minimally-processed pomegranate arils, cv. Wonderful, as affected by $\mathrm{CaCl}$, Ca-lactate and salicylic acid treatments during storage at $5 \pm 1^{\circ} \mathrm{C}(2013$ and 2014 seasons)

\begin{tabular}{|c|c|c|c|c|c|c|c|c|c|c|c|c|}
\hline \multirow{3}{*}{$\begin{array}{l}\text { TSS } \\
\text { Treatments }\end{array}$} & \multicolumn{6}{|c|}{ Season 2013} & \multicolumn{6}{|c|}{ Season 2014} \\
\hline & \multicolumn{6}{|c|}{ Storage period(days) } & \multicolumn{6}{|c|}{ Storage period(days) } \\
\hline & 0 & 3 & 6 & 9 & 12 & Mean & 0 & 3 & 6 & 9 & 12 & Mean \\
\hline Control & $13.63 \mathrm{jk}$ & $13.73 \mathrm{jk}$ & $14.37 \mathrm{gk}$ & $14.60 \mathrm{~g}-\mathrm{k}$ & $15.17 \mathrm{~b}-\mathrm{i}$ & $14.30 \mathrm{~B}$ & $13.83 \mathrm{~h}$ & $13.77 \mathrm{~h}$ & $14.90 \mathrm{f}$ & $15.13 c-f$ & $15.33 \mathrm{~b}-\mathrm{f}$ & $14.59 \mathrm{~B}$ \\
\hline $\mathrm{CaCl}_{2}, 0.5 \%$ & $13.63 \mathrm{jk}$ & $13.73 \mathrm{jk}$ & $15.13 \mathrm{c}-\mathrm{i}$ & 16.03 ae & $16.20 a-d$ & $14.95 \mathrm{AB}$ & $13.83 \mathrm{~h}$ & $13.83 \mathrm{~h}$ & $15.07 \mathrm{ef}$ & $16.07 \mathrm{abc}$ & $16.33 a$ & $15.03 \mathrm{AB}$ \\
\hline $\mathrm{CaCl}_{2}, 1 \%$ & $13.63 \mathrm{jk}$ & $14.13 \mathrm{~h}-\mathrm{k}$ & $14.17 \mathrm{~h}-\mathrm{k}$ & $16.37 \mathrm{abc}$ & $16.77 \mathrm{a}$ & $15.01 \mathrm{~A}$ & $13.83 \mathrm{~h}$ & $13.93 \mathrm{gh}$ & $14.80 \mathrm{fg}$ & $16.03 \mathrm{a}-\mathrm{d}$ & $16.37 \mathrm{a}$ & $14.99 \mathrm{AB}$ \\
\hline Ca-lactate, $0.5 \%$ & $13.63 \mathrm{jk}$ & $14.07 \mathrm{ijk}$ & 14.07 ijk & $15.17 \mathrm{~b}-\mathrm{i}$ & $15.97 \mathrm{a}-\mathrm{f}$ & $14.54 \mathrm{AB}$ & $13.83 \mathrm{~h}$ & $13.90 \mathrm{gh}$ & $14.53 \mathrm{fgh}$ & $15.03 \mathrm{f}$ & 16.00 ae & $14.66 \mathrm{AB}$ \\
\hline Ca-lactate, $1 \%$ & $13.63 \mathrm{jk}$ & $14.70 f_{-j}$ & $14.83 e-j$ & $15.37 \mathrm{~b}-\mathrm{h}$ & $16.40 \mathrm{ab}$ & $14.99 \mathrm{AB}$ & $13.83 \mathrm{~h}$ & 14.67 fgh & $14.97 \mathrm{f}$ & $14.97 \mathrm{f}$ & $16.30 \mathrm{a}$ & $14.95 \mathrm{AB}$ \\
\hline Salicylicacid, $1 \mathrm{mM}$ & $13.63 \mathrm{jk}$ & $14.67 \mathrm{~g}-\mathrm{k}$ & $15.10 \mathrm{~d}-\mathrm{i}$ & $15.10 \mathrm{~d}-\mathrm{i}$ & $15.93 \mathrm{a}-\mathrm{f}$ & $14.89 \mathrm{AB}$ & $13.83 \mathrm{~h}$ & 14.53 fogh & $14.50 \mathrm{fgh}$ & 15.10def & $16.27 \mathrm{ab}$ & $14.85 \mathrm{AB}$ \\
\hline Salicylicacid, $2 \mathrm{mM}$ & $13.63 \mathrm{jk}$ & $14.17 \mathrm{~h}-\mathrm{k}$ & $15.50 \mathrm{bg}$ & $16.07 \mathrm{a}-\mathrm{e}$ & $16.13 a-d$ & $15.10 \mathrm{~A}$ & $13.83 \mathrm{~h}$ & $13.93 \mathrm{gh}$ & $15.43 \mathrm{a}-\mathrm{f}$ & 16.00 aee & $16.30 \mathrm{a}$ & $15.10 \mathrm{~A}$ \\
\hline Mean & $13.63 \mathrm{C}$ & $14.18 \mathrm{~B}$ & $14.74 \mathrm{~B}^{\circ}$ & $15.53 \mathrm{~A}$ & $16.08 \mathrm{~A}$ & & $13.83 \mathrm{D}$ & $14.08 \mathrm{D}$ & $14.89 \mathrm{C}$ & $15.48 \mathrm{~B}$ & $16.13 \mathrm{~A}$ & \\
\hline
\end{tabular}

Note: Differentlettersindicatesignificantly different valuesby ANOVA followed by Duncan testat $\mathrm{P} \leq 0.05$ (small letters refer tovalues recorded in each season, different capital letters refer to mean values)

Table2.Acidity (\%) of minimally-processed pomegranate arils, cr Wonderful, as affected by $\mathrm{CaCl}, \mathrm{Ca}$-lactateand salicylicacid treatments duringstorage at $5 \pm 1{ }^{\circ} \mathrm{C}(2013$ and 2014 seasons $)$

\begin{tabular}{|c|c|c|c|c|c|c|c|c|c|c|c|c|}
\hline \multirow{3}{*}{$\begin{array}{l}\text { Acidity } \\
\text { Treatments }\end{array}$} & \multicolumn{6}{|c|}{ Season 2013} & \multicolumn{6}{|c|}{ Season 2014} \\
\hline & \multicolumn{6}{|c|}{ Storage period(days) } & \multicolumn{6}{|c|}{ Storage period(days) } \\
\hline & 0 & 3 & 6 & 9 & 12 & Mean & 0 & 3 & 6 & 9 & 12 & Mean \\
\hline Control & $1.20 \mathrm{a}$ & $0.93 \mathrm{~b}$ & $0.60 \mathrm{~d}-\mathrm{g}$ & $0.60 \mathrm{~d}-\mathrm{g}$ & $0.50 \mathrm{gh}$ & $0.77 \mathrm{ABC}$ & $1.33 \mathrm{a}$ & $1.1 \mathrm{~b}$ & $0.63 \mathrm{def}$ & $0.60 \mathrm{ef}$ & 0.50 efg & $0.83 \mathrm{~A}$ \\
\hline $\mathrm{CaCl}_{2}, 0.5 \%$ & $1.20 \mathrm{a}$ & $0.73 c$ & $0.60 \mathrm{dg}$ & $0.50 \mathrm{gh}$ & $0.43 \mathrm{~h}$ & $0.69 \mathrm{D}$ & $1.33 \mathrm{a}$ & $0.67 \mathrm{de}$ & $0.63 \mathrm{def}$ & $0.47 \mathrm{fg}$ & $0.47 \mathrm{fg}$ & $0.71 \mathrm{C}$ \\
\hline $\mathrm{CaCl}_{2}, 1 \%$ & $1.20 \mathrm{a}$ & $0.87 \mathrm{~b}$ & $0.67 \mathrm{cde}$ & $0.60 \mathrm{~d}-\mathrm{g}$ & 0.53 fogh & $0.77 \mathrm{AB}$ & $1.33 \mathrm{a}$ & $0.60 \mathrm{ef}$ & $0.60 \mathrm{ef}$ & 0.57 efg & $0.53 \mathrm{efg}$ & $0.73 \mathrm{BC}$ \\
\hline Ca-lactate, $0.5 \%$ & $1.20 \mathrm{a}$ & $0.63 \mathrm{cf}$ & $0.60 \mathrm{dg}$ & $0.60 \mathrm{~d}-\mathrm{g}$ & 0.53 fogh & $0.71 \mathrm{CD}$ & $1.33 \mathrm{a}$ & $0.63 \mathrm{def}$ & $0.57 \mathrm{efg}$ & $0.47 \mathrm{fg}$ & $0.40 \mathrm{~g}$ & $0.68 \mathrm{C}$ \\
\hline Ca-lactate, 1\% & $1.20 \mathrm{a}$ & $0.70 \mathrm{~cd}$ & $0.67 \mathrm{cde}$ & $0.66 \mathrm{cde}$ & 0.53 fogh & $0.75 \mathrm{ABC}$ & $1.33 \mathrm{a}$ & $0.87 \mathrm{c}$ & $0.67 \mathrm{de}$ & $0.60 \mathrm{ef}$ & $0.57 \mathrm{efg}$ & $0.81 \mathrm{AB}$ \\
\hline Salicylicacid, $1 \mathrm{mM}$ & $1.20 \mathrm{a}$ & $0.70 \mathrm{~cd}$ & $0.60 \mathrm{~d}-\mathrm{g}$ & 0.57 efg & 0.53 fogh & $0.72 \mathrm{BCD}$ & $1.33 \mathrm{a}$ & $0.63 \mathrm{def}$ & $0.57 \mathrm{efg}$ & $0.53 \mathrm{efg}$ & $0.47 \mathrm{fg}$ & $0.71 \mathrm{C}$ \\
\hline Salicylicacid, $2 \mathrm{mM}$ & $1.20 \mathrm{a}$ & $0.87 \mathrm{~b}$ & $0.70 \mathrm{~cd}$ & $0.60 \mathrm{~d}-\mathrm{g}$ & $0.57 \mathrm{efg}$ & $0.79 \mathrm{~A}$ & $1.33 \mathrm{a}$ & $0.80 \mathrm{~cd}$ & $0.63 \mathrm{def}$ & $0.53 \mathrm{efg}$ & $0.47 \mathrm{fg}$ & $0.75 \mathrm{ABC}$ \\
\hline Mean & $1.20 \mathrm{~A}$ & $0.78 \mathrm{~B}$ & $0.63 \mathrm{C}$ & $0.59 \mathrm{C}$ & $0.52 \mathrm{D}$ & & $1.33 \mathrm{~A}$ & $0.75 \mathrm{~B}$ & $0.61 \mathrm{C}$ & $0.54 \mathrm{CD}$ & $0.49 \mathrm{D}$ & \\
\hline
\end{tabular}

Note: Differentlettersindicatesignificantly differentvaluesby ANOVAfollowedbyDuncan testat $\mathrm{P} \leq 0.05$ (small letters refer tovalues recorded in each season, different capital letters refer to mean values)

Table 3. Ascorbic acid (Vitamin C) content ( $\mathrm{mg} / 100 \mathrm{ml})$ of minimally-processed pomegranate arils, $\mathrm{cv}$ Wonderful, as affected by $\mathrm{CaCl}$, Ca-lactate and salicylic acid treatments during storage at $\underline{5 \pm 1^{\circ} \mathrm{C}(2013 \text { and2014seasons })}$

\begin{tabular}{|c|c|c|c|c|c|c|c|c|c|c|c|c|}
\hline \multirow{3}{*}{ Treatments } & \multicolumn{6}{|c|}{ Season 2013} & \multicolumn{6}{|c|}{ Season 2014} \\
\hline & \multicolumn{6}{|c|}{ Storage period(days) } & \multicolumn{6}{|c|}{ Storage period(days) } \\
\hline & 0 & 3 & 6 & 9 & 12 & Mean & 0 & 3 & 6 & 9 & 12 & Mean \\
\hline Control & $4.80 \mathrm{a}$ & $4.57 \mathrm{ab}$ & $3.67 \mathrm{~d}-\mathrm{h}$ & $2.40 \mathrm{mno}$ & 2.230 & $3.53 \mathrm{BC}$ & $5.03 a$ & $4.467 b$ & $3.70 \mathrm{~cd}$ & 2.57ghi & $2.03 j$ & $3.56 \mathrm{~B}$ \\
\hline $\mathrm{CaCl} 2,0.5 \%$ & $4.80 \mathrm{a}$ & $4.23 b c$ & $3.63 \mathrm{e}-\mathrm{i}$ & $2.77 \mathrm{k}-\mathrm{n}$ & 2.27 no & $3.54 \mathrm{BC}$ & $5.03 a$ & $4.33 b$ & $3.70 \mathrm{~cd}$ & $2.70 \mathrm{f}-\mathrm{i}$ & $2.50 \mathrm{hi}$ & $3.65 \mathrm{AB}$ \\
\hline $\mathrm{CaCl} 2,1 \%$ & $4.80 \mathrm{a}$ & $4.33 \mathrm{ab}$ & $3.77 c-f$ & 3.13 ijk & $2.87 \mathrm{j}-\mathrm{m}$ & $3.78 \mathrm{AB}$ & $5.03 a$ & $4.40 \mathrm{~b}$ & $3.50 \mathrm{de}$ & $2.87 \mathrm{fgh}$ & $2.67 \mathrm{f}-\mathrm{i}$ & $3.69 \mathrm{AB}$ \\
\hline Ca-lactate, $0.5 \%$ & $4.80 \mathrm{a}$ & $3.63 \mathrm{e}-\mathrm{i}$ & $3.37 \mathrm{f}-\mathrm{j}$ & $2.87 \mathrm{j}-\mathrm{m}$ & $2.80 \mathrm{klm}$ & $3.49 \mathrm{C}$ & $5.03 \mathrm{a}$ & $4.20 \mathrm{~b}$ & $3.67 \mathrm{~cd}$ & $2.80 \mathrm{fgh}$ & $2.67 \mathrm{f}-\mathrm{i}$ & $3.67 \mathrm{AB}$ \\
\hline Ca-lactate, $1 \%$ & $4.80 \mathrm{a}$ & $4.16 \mathrm{bcd}$ & 4.10be & $3.17 \mathrm{~h}-\mathrm{k}$ & $2.571-o$ & $3.76 \mathrm{ABC}$ & $5.03 \mathrm{a}$ & $4.30 \mathrm{~b}$ & $3.73 \mathrm{~cd}$ & $3.07 \mathrm{ef}$ & 2.57ghi & $3.74 \mathrm{AB}$ \\
\hline Salicylic acid, $1 \mathrm{mM}$ & $4.80 \mathrm{a}$ & $4.50 \mathrm{ab}$ & $3.73 c-g$ & $3.17 \mathrm{~h}-\mathrm{k}$ & $2.93 \mathrm{jkl}$ & $3.82 \mathrm{~A}$ & $5.03 a$ & $4.47 \mathrm{~b}$ & $4.07 \mathrm{bc}$ & $3.10 \mathrm{ef}$ & $2.33 \mathrm{ij}$ & $3.80 \mathrm{~A}$ \\
\hline Salicylic acid, $2 \mathrm{mM}$ & $4.80 \mathrm{a}$ & $4.33 \mathrm{ab}$ & 4.07 be & $3.23 \mathrm{~g}-\mathrm{k}$ & $2.57 \mathrm{l}-\mathrm{o}$ & $3.80 \mathrm{AB}$ & $5.03 \mathrm{a}$ & $4.27 b$ & $4.07 \mathrm{bc}$ & $2.97 \mathrm{fg}$ & $2.50 \mathrm{hi}$ & $3.77 \mathrm{AB}$ \\
\hline Mean & $4.80 \mathrm{~A}$ & $4.25 B$ & $3.76 \mathrm{C}$ & $2.96 \mathrm{D}$ & $2.61 \mathrm{E}$ & & $5.03 \mathrm{~A}$ & $4.35 \mathrm{~B}$ & $3.78 \mathrm{C}$ & $2.87 \mathrm{D}$ & $2.47 \mathrm{E}$ & \\
\hline
\end{tabular}

Note: Differentlettersindicatesignificantly different valuesby ANOVAfollowed by Duncantestat $\mathrm{P} \leq 0.05$ (small letters refer tovalues recorded in each season, different capital letters refertomean values)

Table 4. Anthocyanin content $(\mathrm{mg} / 100 \mathrm{ml})$ of minimally-processed pomegranate arils, cv Wonderful, as affected by $\mathrm{CaCl}_{2}$, Ca-lactate and salicylic acid treatments during storage at $5 \pm 1^{\circ} \mathrm{C}$ (2013 and 2014seasons)

\begin{tabular}{|c|c|c|c|c|c|c|c|c|c|c|c|c|}
\hline \multirow{3}{*}{ Treatments } & \multicolumn{6}{|c|}{ Season 2013} & \multicolumn{6}{|c|}{ Season 2014} \\
\hline & \multicolumn{6}{|c|}{ Storage period(days) } & \multicolumn{6}{|c|}{ Storage period(days) } \\
\hline & $\overline{0}$ & 3 & 6 & 9 & 12 & Mean & 0 & 3 & 6 & 9 & 12 & Mean \\
\hline Control & $12.59 a$ & $10.68 \mathrm{bcd}$ & $9.85 \mathrm{cde}$ & $6.49 \mathrm{lmn}$ & $5.19 \mathrm{op}$ & $8.96 \mathrm{~A}$ & $13.20 \mathrm{a}$ & $9.46 \mathrm{c}-\mathrm{h}$ & $8.77 \mathrm{f}-\mathrm{i}$ & $7.19 \mathrm{jkl}$ & $6.26 \mathrm{lmn}$ & $8.98 \mathrm{BC}$ \\
\hline $\mathrm{CaCl}_{2}, 0.5 \%$ & $12.59 a$ & $9.18 \mathrm{e}-\mathrm{h}$ & $7.98 \mathrm{~h}-\mathrm{k}$ & $7.00 \mathrm{klm}$ & 5.45 nop & $8.44 \mathrm{~A}$ & $13.20 \mathrm{a}$ & $10.20 \mathrm{~b}-\mathrm{f}$ & $9.84 \mathrm{c}-\mathrm{g}$ & $8.99 \mathrm{e}-\mathrm{h}$ & 5.06 no & $9.45 \mathrm{ABC}$ \\
\hline $\mathrm{CaCl}, 1 \%$ & $12.59 \mathrm{a}$ & $9.64 \mathrm{~d}-\mathrm{g}$ & 8.48ghi & $6.84 \mathrm{klm}$ & $5.26 \mathrm{op}$ & $8.56 \mathrm{~A}$ & $13.20 \mathrm{a}$ & $10.32 \mathrm{~b}-\mathrm{e}$ & $9.48 c-h$ & $8.71 \mathrm{f}_{-j}$ & $5.47 \mathrm{mno}$ & $9.44 \mathrm{ABC}$ \\
\hline Ca-lactate, $0.5 \%$ & $12.59 a$ & $11.41 \mathrm{ab}$ & 8.29 hij & $7.14 \mathrm{jkl}$ & 5.53 nop & $8.99 \mathrm{~A}$ & $13.20 \mathrm{a}$ & $9.37 \mathrm{~d}-\mathrm{h}$ & $7.97 \mathrm{~h}-\mathrm{k}$ & $7.36 \mathrm{i}-1$ & $5.32 \mathrm{mno}$ & $8.64 \mathrm{C}$ \\
\hline Ca-lactate, $1 \%$ & $12.59 \mathrm{a}$ & $10.93 b c$ & $8.33 \mathrm{hij}$ & $6.91 \mathrm{klm}$ & 6.231-o & $8.99 \mathrm{~A}$ & $13.20 \mathrm{a}$ & $11.55 \mathrm{~b}$ & $9.15 \mathrm{~d}-\mathrm{h}$ & $8.84 \mathrm{e}-\mathrm{i}$ & $6.55 \mathrm{k}-\mathrm{n}$ & $9.89 \mathrm{~A}$ \\
\hline Salicylic acid, $1 \mathrm{mM}$ & $12.59 a$ & $9.72 \mathrm{def}$ & $8.61 \mathrm{f}-\mathrm{i}$ & $6.291 \mathrm{lo}$ & $4.86 \mathrm{p}$ & $8.42 \mathrm{~A}$ & $13.20 \mathrm{a}$ & $10.98 \mathrm{bc}$ & $8.34 \mathrm{~g}-\mathrm{j}$ & $6.33 \mathrm{lmn}$ & 4.710 & $8.71 \mathrm{BC}$ \\
\hline Salicylicacid, $2 \mathrm{mM}$ & $12.59 \mathrm{a}$ & $9.73 c-f$ & 8.27 hij & $7.83 \mathrm{ijk}$ & $5.83 \mathrm{~m}-\mathrm{p}$ & $8.85 \mathrm{~A}$ & $13.20 \mathrm{a}$ & $10.67 \mathrm{bcd}$ & $9.19 \mathrm{~d}-\mathrm{h}$ & $7.96 \mathrm{~h}-\mathrm{k}$ & $6.63 \mathrm{klm}$ & $9.53 \mathrm{AB}$ \\
\hline Mean & $12.59 \mathrm{~A}$ & $10.18 \mathrm{~B}$ & $8.54 \mathrm{C}$ & $6.93 \mathrm{D}$ & $5.48 \mathrm{E}$ & & $13.20 \mathrm{~A}$ & $10.36 \mathrm{~B}$ & $8.96 \mathrm{C}$ & $7.91 \mathrm{D}$ & $5.71 \mathrm{E}$ & \\
\hline
\end{tabular}

Note: Differentlettersindicatesignificantly differentvaluesby ANOVA followed by Duncan testat $\mathrm{P} \leq 0.05$ (small letters refer tovalues recorded in each season, different capital letters refer to mean values)

Table 5. Firmness $\left(\mathrm{gcm}^{-2}\right)$ of minimally-processed pomegranate arils, $\mathrm{cv}$ Wonderful, as affected by $\mathrm{CaCl}, \mathrm{Ca}$-lactate and salicylic acid treatments duringstorage at $5 \pm 1^{\circ} \mathrm{C}(2013$ and 2014 seasons $)$

\begin{tabular}{|c|c|c|c|c|c|c|c|c|c|c|c|c|}
\hline \multirow{3}{*}{ Treatments } & \multicolumn{6}{|c|}{ Season 2013} & \multicolumn{6}{|c|}{ Season 2014} \\
\hline & \multicolumn{6}{|c|}{ Storage period(days) } & \multicolumn{6}{|c|}{ Storage period(days) } \\
\hline & 0 & 3 & 6 & 9 & 12 & Mean & 0 & 3 & 6 & 9 & 12 & Mean \\
\hline Control & $15.67 \mathrm{a}$ & $11.67 \mathrm{c}-\mathrm{f}$ & $8.34 \mathrm{~h}-1$ & $7.001-0$ & 5.330 & $9.60 \mathrm{D}$ & $13.67 \mathrm{a}$ & $11.00 \mathrm{c}-\mathrm{g}$ & $8.33 \mathrm{i}-1$ & $7.00 \mathrm{lmn}$ & $4.67 \mathrm{o}$ & $8.93 \mathrm{C}$ \\
\hline $\mathrm{CaCl}_{2}, 0.5 \%$ & $15.67 \mathrm{a}$ & $13.33 b c$ & $11.00 \mathrm{~d}-\mathrm{g}$ & $9.00 \mathrm{~g}-1$ & $7.33 \mathrm{k}-\mathrm{o}$ & $11.27 \mathrm{AB}$ & $13.67 \mathrm{a}$ & $12.00 \mathrm{a}-\mathrm{d}$ & $10.33 \mathrm{~d}-\mathrm{h}$ & $8.67 \mathrm{~h}-\mathrm{l}$ & $7.67 \mathrm{j}-\mathrm{m}$ & $10.47 \mathrm{~B}$ \\
\hline $\mathrm{CaCl}_{2} 1 \%$ & $15.67 \mathrm{a}$ & $14.00 \mathrm{ab}$ & $11.67 \mathrm{c}-\mathrm{f}$ & $9.00 \mathrm{~g}-\mathrm{l}$ & $7.33 \mathrm{k}-\mathrm{o}$ & $11.53 \mathrm{~A}$ & $13.67 \mathrm{a}$ & $13.00 \mathrm{ab}$ & $12.33 a b c$ & $11.33 b-f$ & $9.67 \mathrm{f}-\mathrm{i}$ & $12.00 \mathrm{~A}$ \\
\hline Ca-lactate, $0.5 \%$ & $15.67 \mathrm{a}$ & $12.67 \mathrm{bcd}$ & $10.00 \mathrm{e}-\mathrm{i}$ & $8.33 \mathrm{~h}-1$ & $7.00 \mathrm{l}-\mathrm{o}$ & $10.73 \mathrm{ABC}$ & $13.67 \mathrm{a}$ & $11.67 \mathrm{~b}-\mathrm{e}$ & $10.00 \mathrm{ei}$ & $9.00 \mathrm{~h}-\mathrm{k}$ & $7.33 \mathrm{k}-\mathrm{n}$ & $10.33 \mathrm{~B}$ \\
\hline Ca-lactate, $1 \%$ & $15.67 \mathrm{a}$ & $12.67 \mathrm{bcd}$ & $10.33 \mathrm{e}-\mathrm{h}$ & $8.00 \mathrm{i}-\mathrm{m}$ & $6.00 \mathrm{mno}$ & 10.53A-D & $13.67 \mathrm{a}$ & $12.00 \mathrm{a}-\mathrm{d}$ & $10.33 \mathrm{~d}-\mathrm{h}$ & $9.33 \mathrm{~g}-\mathrm{j}$ & $7.33 \mathrm{k}-\mathrm{n}$ & $10.53 \mathrm{~B}$ \\
\hline Salicylicacid, $1 \mathrm{mM}$ & $15.67 \mathrm{a}$ & $12.00 \mathrm{~b}-\mathrm{e}$ & $9.67 f_{-j}$ & $7.67 j-n$ & $5.67 \mathrm{no}$ & $10.13 \mathrm{CD}$ & $13.67 \mathrm{a}$ & $11.67 \mathrm{~b}-\mathrm{e}$ & $9.33 \mathrm{~g}-j$ & $7.33 \mathrm{k}-\mathrm{n}$ & 5.67 no & $9.53 \mathrm{BC}$ \\
\hline Salicylicacid, $2 \mathrm{mM}$ & $15.67 \mathrm{a}$ & $13.00 \mathrm{bcd}$ & $9.34 \mathrm{~g}-\mathrm{k}$ & $7.33 \mathrm{k}-\mathrm{o}$ & $6.00 \mathrm{no}$ & $10.27 \mathrm{BCD}$ & $13.67 \mathrm{a}$ & $11.33 b-f$ & $9.33 \mathrm{~g}-j$ & $7.67 \mathrm{j}-\mathrm{m}$ & $6.00 \mathrm{mno}$ & $9.60 \mathrm{BC}$ \\
\hline Mean & $15.67 \mathrm{~A}$ & $12.76 \mathrm{~B}$ & $10.05 \mathrm{C}$ & $8.05 \mathrm{D}$ & $6.38 \mathrm{E}$ & & $13.67 \mathrm{~A}$ & $11.81 \mathrm{~B}$ & $10.00 \mathrm{C}$ & $8.62 \mathrm{D}$ & $6.90 \mathrm{E}$ & \\
\hline
\end{tabular}

Note: Differentlettersindicatesignificantly different values by ANOVA followed by Duncan testat $\mathrm{P} \leq 0.05$ (small letters refer tovalues recorder in each season, different capital letters refertomean values) 
512

fruits (Ding et al., 2007), or $\mathrm{CaCl}_{2}$ on strawberry (Biten Court De Souza et al., 1999)

\section{Ascorbic acid (Vitamin C) content}

Ascorbic acid is an important nutrient quality parameter. However, compared to other nutrients, it is very sensitive to degradation due to its oxidation during food processing and storage (Veltman et al., 2000). Pomegranates are low in vitamin $\mathrm{C}$, its content ranging from 0.49 to $30 \mathrm{mg}$ per $100 \mathrm{~g}$ of juice, depending on cultivar (Hussein and Hussein, 1972; Küpper, 1995). Table 3 shows that, in both seasons, vitamin $C$ content significantly decreased with the increase of storage time, in accordance with O'Grady et al. (2014) who observed that ascorbic acid concentration reduced over time in 'Ruby' arils stored at $1{ }^{\circ} \mathrm{C}, 4^{\circ} \mathrm{C}$ and $8{ }^{\circ} \mathrm{C}$ for 7 days. With few exceptions, $\mathrm{SA}$ at $1 \mathrm{mM}$ gave the highest values at each storage period of both the seasons, determining also the highest mean values, with significant differences with the control. Also SA at $2 \mathrm{mM}$ maintained relatively high values of vitamin $\mathrm{C}$; in this context, Sayyari et al. (2009) found that SA at $2 \mathrm{mM}$ reduced the decline rate in ascorbic acid content, compared to control fruit.

Results are consistent with the finding of Sayyari et al. (2011) who found that ascorbic acid losses were about 50\% in control fruits at the end of storage time, and significant increases were observed for 0.5 and $1 \mathrm{mM}$ acetylsalicylic acid. This could be attributed to the effects of acetylsalicylic acid on promoting the ascorbate-glutathione cycle (Wang et al., 2006) and improving levels of antioxidant components such as ascorbic acid (Huang et al., 2008). Differently from this study, Mirdehghan and Ghotbi (2014) reported that pomegranate fruits treated with $2 \mathrm{mM}$ SA had the lowest ascorbic acid content, although differences between other treatments and with the untreated fruits were not significant,

Lowest mean values of ascorbic acid were always obtained with control arils, although the differences with the treated ones were only occasionally significant. Aarabi et al. (2008) also investigated the concentration of ascorbic acid in selected pomegranate juices during storage at $4{ }^{\circ} \mathrm{C}$ for 60 days, and reported $100 \%$ loss of initial ascorbic acid concentration after 15 days at $4{ }^{\circ} \mathrm{C}$. Similarly, a significant loss in vitamin C concentration was observed in pomegranate fruit 'Wonderful' stored at $5^{\circ} \mathrm{C}$ and $7.5^{\circ} \mathrm{C}$ for 5 months (Arendse et al., 2014). A decrease in vitamin $\mathrm{C}$ may be related to the irreversible oxidation of dehydro-L-ascorbic acid (DHAA) to 2,3-diketo$\mathrm{L}$-gulonic acid (Coultate, 2007). Furthermore, ascorbic acid is affected (and its activity is reduced) by the presence of oxygen, alkalinity and high temperatures (Coultate, 2007).

\section{Anthocyanins content}

Anthocyanins are responsible for the desirable red color of pomegranate juices, as well as many other red-colored fruit juices ( $\mathrm{Li}$ et al., 2010). Generally, anthocyanins are labile compounds and are easily susceptible to degradation (Mphahlele et al., 2014).

Table 4 shows that anthocyanin content significantly decreased throughout the storage time in both the seasons, irrespective to the treatments. No significant mean difference was observed in the first season among the different treatments and control, while in the second season arils treated with $1 \%$ Ca-lactate had the highest anthocyanin content, significantly different from control arils and the ones treated with $0.5 \% \mathrm{Ca}^{-}$ lactate. SA at $2 \mathrm{mM}$ maintained the highest value of anthocyanin after 12 days of storage, in accordance with the results obtained by Sayyari et al. (2011) who showed that acetyl salicylic acid maintains high levels of bioactive compounds, such as total anthocyanins.

These data show that anthocyanin content is significantly influenced by storage time, but treatments have a nonsignificant effect on anthocyanin content on minimally processed arils. This result is in accordance with those of Caleb et al. (2013) on minimally processed pomegranates, as they reported a significant effect of storage duration on the total anthocyanin content, with a general trend of a decrease in total anthocyanin content as the storage time increased. Also Arendse et al. (2014) reported a decline in total anthocyanin concentration of 'Wonderful' pomegranate after cold storage. The decrease in phenolic concentration, including anthocyanins, in pomegranates could be attributed to the change of enzyme activities resulting to phenolic degradation (Fawole and Opara, 2013). Furthermore, loss of anthocyanins could be attributed to many other factors, such as $\mathrm{pH}$ and acidity, phenolic compounds, sugars and sugar degradation products, oxygen, ascorbic acid, fruit maturity and thawing time (Withy et al., 1993; García-Viguera et al.,1998).

\section{Firmness}

As shown in Table 5, a significant reduction of arils firmness within the storage period was observed in both the investigated seasons and regardless to the treatments. As for the different treatments, calcium chloride treatments at both the tested concentrations $(0.5 \%$ and $1 \%)$ maintained the highest arils firmness, with significant differences with the untreated arils. In both seasons, at every storage period, untreated arils had the lowest firmness, with values significantly different from the calcium-treated ones. In accordance with these results, Aguayo et al. (2012) reported that $\mathrm{CaCl}_{2}$ treatments kept a better firmness of pomegranates fruits than control arils. It is well known that firmness and resistance to softening can be increased by the addition of calcium, due to the stabilization of the membrane systems and the formation of Ca-pectates which increase the rigidity of the middle lamella and cell wall, as well as the cell cohesion (White and Broadley, 2003). Manganaris et al. (2007) suggested the $\mathrm{CaCl}_{2}$ immersion as potential postharvest treatment for whole peaches, since it increases tissue firmness and reduces susceptibility to physiological disorders. Moreover, the use of $\mathrm{CaCl}_{2}$ dips is recommended as a low cost-effective method for extending the storage life of arils pomegranate (Aguayo et al., 2012). Mirdehghan and Ghotbi (2014) reported that calcium maintains the cell wall structure in fruit by interacting with the pectic acid in the cell walls to form calcium pectate. In addition, Garcia et al. (1996) and Picchioni et al. (1998) indicated that postharvest calcium application maintains cell turgor, membrane integrity, tissue firmness, and delays membrane lipid catabolism, extending storage life of fresh fruits and reducing physiological disorders. El-Kassas et al. (1995) also obtained satisfactory results on pomegranate with pre- and postharvest $\mathrm{CaCl}_{2}$ treatments.

As for the treatments with SA, no significant difference in aril firmness with the control was observed at each storage period, in accordance with what observed on kiwifruits treated with acetyl salicylic acid (Sayyari et al., 2011). 
Table6.Color ( $\mathrm{L}^{*}$ value) of minimally-processed pomegranatearils,cv Wonderful, as affected by $\mathrm{CaCl}_{2}$, Ca-lactate and salicylic acid trearmentsduringstorage at $5 \pm 1^{\circ} \mathrm{C}(2013$ and 2014 seasons $)$

\begin{tabular}{|c|c|c|c|c|c|c|c|c|c|c|c|c|}
\hline \multirow{3}{*}{ Treatments } & \multicolumn{6}{|c|}{ Season 2013} & \multicolumn{6}{|c|}{ Season 2014} \\
\hline & \multicolumn{6}{|c|}{ Storage period (days) } & \multicolumn{6}{|c|}{ Storage period (days) } \\
\hline & 0 & 3 & 6 & 9 & 12 & Mean & 0 & 3 & 6 & 9 & 12 & Mean \\
\hline Control & $48.50 \mathrm{a}$ & $31.77 \mathrm{~b}-\mathrm{f}$ & $26.67 \mathrm{~d}-\mathrm{h}$ & $23.37 \mathrm{~g}-\mathrm{k}$ & $22.37 \mathrm{gk}$ & $30.53 \mathrm{AB}$ & $49.40 \mathrm{a}$ & $29.57 \mathrm{~cd}$ & $29.50 \mathrm{~cd}$ & $26.87 \mathrm{~d}-\mathrm{g}$ & $24.77 \mathrm{~d}-\mathrm{j}$ & $32.02 \mathrm{~A}$ \\
\hline $\mathrm{CaCl}, 0.5 \%$ & $48.50 \mathrm{a}$ & $32.33 \mathrm{be}$ & $27.00 \mathrm{~d}-\mathrm{h}$ & $20.20 \mathrm{ijk}$ & $18.63 \mathrm{k}$ & $29.33 \mathrm{~B}$ & $49.40 \mathrm{a}$ & $25.90 \mathrm{~d}-\mathrm{h}$ & $24.93 \mathrm{~d}-\mathrm{j}$ & $23.07 \mathrm{e}-\mathrm{j}$ & $21.53 \mathrm{~g}-j$ & $28.97 \mathrm{~A}$ \\
\hline $\mathrm{CaCl}_{2}, 1 \%$ & $48.50 \mathrm{a}$ & $34.63 \mathrm{bc}$ & $27.74 \mathrm{dg}$ & $23.00 \mathrm{~g}-\mathrm{k}$ & $21.33 \mathrm{~h}-\mathrm{k}$ & $31.04 \mathrm{AB}$ & $49.40 \mathrm{a}$ & $27.63 \mathrm{c}-\mathrm{f}$ & $27.60 \mathrm{c}-\mathrm{f}$ & 20.56hij & $19.09 \mathrm{j}$ & $28.86 \mathrm{~A}$ \\
\hline Ca-lactate, $0.5 \%$ & $48.50 \mathrm{a}$ & $30.87 \mathrm{~b}-\mathrm{f}$ & $26.22 \mathrm{e}-\mathrm{i}$ & $25.42 \mathrm{f}_{-j}$ & $23.05 \mathrm{gk}$ & $30.81 \mathrm{AB}$ & $49.40 \mathrm{a}$ & $29.73 c d$ & $27.68 \mathrm{cde}$ & 20.84hij & $19.71 \mathrm{ij}$ & $29.47 \mathrm{~A}$ \\
\hline Ca-lactate, 1\% & $48.50 \mathrm{a}$ & $31.03 \mathrm{~b}-\mathrm{f}$ & $30.47 \mathrm{c}-\mathrm{f}$ & $23.87 \mathrm{~g}-\mathrm{k}$ & $21.35 \mathrm{~h}-\mathrm{k}$ & $31.04 \mathrm{AB}$ & $49.40 \mathrm{a}$ & $29.10 \mathrm{~cd}$ & $25.67 \mathrm{~d}-\mathrm{i}$ & $21.86 \mathrm{e}-\mathrm{j}$ & 20.50hij & $29.31 \mathrm{~A}$ \\
\hline Salicylicacid, $1 \mathrm{mM}$ & $48.50 \mathrm{a}$ & $36.90 \mathrm{~b}$ & $26.43 \mathrm{~d}-\mathrm{i}$ & $21.14 \mathrm{~h}-\mathrm{k}$ & $19.66 \mathrm{jk}$ & $30.53 \mathrm{AB}$ & $49.40 \mathrm{a}$ & $3937 \mathrm{~b}$ & $24.32 \mathrm{~d}-\mathrm{j}$ & $24.03 \mathrm{~d}-\mathrm{j}$ & $21.68 f-j$ & $31.76 \mathrm{~A}$ \\
\hline Salicylicacid, $2 \mathrm{mM}$ & $48.50 \mathrm{a}$ & $32.77 \mathrm{bcd}$ & $31.14 \mathrm{~b}-\mathrm{f}$ & $28.07 \mathrm{~d}-\mathrm{g}$ & $26.48 \mathrm{~d}-\mathrm{i}$ & $33.39 \mathrm{~A}$ & $49.40 \mathrm{a}$ & $33.53 \mathrm{bc}$ & $26.06 \mathrm{~d}-\mathrm{h}$ & $22.93 \mathrm{e}-\mathrm{j}$ & 20.50hij & $30.49 \mathrm{~A}$ \\
\hline Mean & $48.50 \mathrm{~A}$ & $32.90 \mathrm{~B}$ & $27.95 \mathrm{C}$ & $23.58 \mathrm{D}$ & $21.84 \mathrm{D}$ & & $49.40 \mathrm{~A}$ & $30.69 \mathrm{~B}$ & $26.54 \mathrm{C}$ & $22.88 \mathrm{D}$ & $21.11 \mathrm{D}$ & \\
\hline
\end{tabular}

Note:Differentlettersindicatesignificantly differentvaluesby ANOVAfollowed by Duncan testat $\mathrm{P} \leq 0.05$ (small letters refer tovalues recorded ineach season, different capital letters refer to mean values)

Table 7. Hue angel $\left(\mathrm{h}^{\circ}\right)$ values of minimally-processed pomegranate arils, cr Wonderful, as affected by $\mathrm{CaCl}$, Ca-lactate and salicylic acid treatments during storage at $5 \pm 1^{\circ} \mathrm{C}(2013$ and 2014 seasons)

\begin{tabular}{|c|c|c|c|c|c|c|c|c|c|c|c|c|}
\hline \multirow{3}{*}{ Treatments } & \multicolumn{6}{|c|}{ Season 2013} & \multicolumn{6}{|c|}{ Season 2014} \\
\hline & \multicolumn{6}{|c|}{ Storage period (days) } & \multicolumn{6}{|c|}{ Storage period (days) } \\
\hline & 0 & 3 & 6 & 9 & 12 & Mean & 0 & 3 & 6 & 9 & 12 & Mean \\
\hline Control & $37.77 \mathrm{ab}$ & $35.80 \mathrm{a}-\mathrm{f}$ & $32.57 \mathrm{a}-\mathrm{i}$ & $29.63 \mathrm{e}-\mathrm{i}$ & $29.27 \mathrm{f}-\mathrm{i}$ & $33.01 \mathrm{~A}$ & $37.80 \mathrm{a}$ & $34.17 \mathrm{a}-\mathrm{f}$ & $32.80 \mathrm{a}-\mathrm{g}$ & $29.20 \mathrm{f}-\mathrm{j}$ & $27.57 \mathrm{~g}-\mathrm{j}$ & $32.31 \mathrm{~A}$ \\
\hline $\mathrm{CaCl}_{2}, 0.5 \%$ & $37.77 \mathrm{ab}$ & $36.97 \mathrm{a}-\mathrm{d}$ & $31.53 \mathrm{a}-\mathrm{i}$ & $30.73 c-i$ & $26.60 \mathrm{i}$ & $32.72 \mathrm{~A}$ & $37.80 \mathrm{a}$ & $35.50 \mathrm{a}-\mathrm{e}$ & $30.77 \mathrm{e}-\mathrm{i}$ & $27.67 \mathrm{~g}-\mathrm{j}$ & $26.97 \mathrm{hij}$ & $31.74 \mathrm{~A}$ \\
\hline $\mathrm{CaCl}_{2}, 1 \%$ & $37.77 \mathrm{ab}$ & $36.51 \mathrm{a}-\mathrm{e}$ & $33.59 \mathrm{a}-\mathrm{i}$ & $29.72 \mathrm{e}-\mathrm{i}$ & $26.67 \mathrm{i}$ & $32.85 \mathrm{~A}$ & $37.80 \mathrm{a}$ & $36.54 a b c$ & $29.76 \mathrm{f}-\mathrm{i}$ & 26.96hij & $26.53 \mathrm{ij}$ & $31.52 \mathrm{~A}$ \\
\hline Ca-lactate, $0.5 \%$ & $37.77 \mathrm{ab}$ & $35.44 \mathrm{a}-\mathrm{g}$ & $32.01 \mathrm{a}-\mathrm{i}$ & $30.77 \mathrm{~b}-\mathrm{i}$ & $30.06 \mathrm{~d}-\mathrm{i}$ & $33.21 \mathrm{~A}$ & $37.80 \mathrm{a}$ & $36.58 \mathrm{abc}$ & $32.20 \mathrm{~b}-\mathrm{h}$ & $32.61 \mathrm{a}-\mathrm{g}$ & $31.10 \mathrm{~d}-\mathrm{i}$ & $34.06 \mathrm{~A}$ \\
\hline Ca-lactate, $1 \%$ & $37.77 \mathrm{ab}$ & $37.88 \mathrm{a}$ & $31.73 \mathrm{a}-\mathrm{i}$ & $31.05 a-i$ & 28.47 ghi & $33.38 \mathrm{~A}$ & $37.80 \mathrm{a}$ & $34.34 a-f$ & $29.80 \mathrm{f}-\mathrm{i}$ & $29.21 f_{-j}$ & $27.67 \mathrm{~g}-\mathrm{j}$ & $31.76 \mathrm{~A}$ \\
\hline Salicylic acid, $1 \mathrm{mM}$ & $37.77 \mathrm{ab}$ & $33.83 \mathrm{a}-\mathrm{h}$ & $36.53 \mathrm{a}-\mathrm{e}$ & $28.83 \mathrm{f}-\mathrm{i}$ & $28.16 \mathrm{hi}$ & $33.02 \mathrm{~A}$ & $37.80 \mathrm{a}$ & $32.03 \mathrm{~b}-\mathrm{h}$ & $30.63 \mathrm{e}-\mathrm{i}$ & $37.17 \mathrm{ab}$ & $28.26 \mathrm{~g}-j$ & $33.18 \mathrm{~A}$ \\
\hline Salicylic acid, $2 \mathrm{mM}$ & $37.77 \mathrm{ab}$ & $37.18 \mathrm{abc}$ & $34.90 \mathrm{a}-\mathrm{h}$ & $32.39 \mathrm{a}-\mathrm{i}$ & 28.53 ghi & $34.15 \mathrm{~A}$ & $37.80 \mathrm{a}$ & $36.32 \mathrm{a}-\mathrm{d}$ & $31.76 \mathrm{c}-\mathrm{i}$ & $30.82 \mathrm{e}-\mathrm{i}$ & $24.23 j$ & $32.25 \mathrm{~A}$ \\
\hline Mean & $37.77 \mathrm{AB}$ & $36.23 \mathrm{AB}$ & $33.27 \mathrm{BC}$ & $30.45 \mathrm{CD}$ & $28.25 \mathrm{D}$ & & $37.80 \mathrm{~A}$ & $35.07 \mathrm{~B}$ & $31.10 \mathrm{C}$ & $30.52 \mathrm{C}$ & $27.47 \mathrm{D}$ & \\
\hline
\end{tabular}

Note:Differentlettersindicatesignificantly different values by ANOVAfollowedby Duncan testat $\mathrm{P} \leq 0.05$ (small letters refer tovalues recordedineach season, differentcapital letters refer to mean values)

Table 8. Microbial count of fungi/yeasts ( $\left.\log \mathrm{CFU} \mathrm{g}^{-1}\right)$ of minimally-processed pomegranate arils, cv Wonderful, as affected by $\mathrm{CaCl}_{2}$, Ca-lactate and salicylic acid treatments during storage at $\underline{5 \pm 1^{\circ} \mathrm{C}(2013 \text { and } 2014 \text { seasons) }}$

\begin{tabular}{|c|c|c|c|c|c|c|c|c|}
\hline \multirow{3}{*}{ Treatments } & \multicolumn{4}{|c|}{ Season 2013} & \multicolumn{4}{|c|}{ Season 2014} \\
\hline & \multicolumn{4}{|c|}{ Storage period (days) } & \multicolumn{4}{|c|}{ Storage period (days) } \\
\hline & 0 & 6 & 12 & Mean & 0 & 6 & 12 & Mean \\
\hline Control & $2.50 \mathrm{a}$ & $2.50 \mathrm{a}$ & $2.57 \mathrm{a}$ & $2.52 \mathrm{~A}$ & $2.50 \mathrm{a}$ & $2.50 \mathrm{a}$ & $2.50 \mathrm{a}$ & $2.50 \mathrm{~A}$ \\
\hline $\mathrm{CaCl}_{2}, 0.5 \%$ & $1.50 \mathrm{~g}$ & $1.73 \mathrm{f}$ & $2.27 \mathrm{c}$ & $1.83 \mathrm{E}$ & $1.47 \mathrm{~g}$ & $1.80 \mathrm{e}$ & $2.13 \mathrm{~d}$ & $1.80 \mathrm{E}$ \\
\hline $\mathrm{CaCl}_{2}, 1 \%$ & $2.03 \mathrm{e}^{\circ}$ & $2.10 \mathrm{de}$ & $2.17 \mathrm{~d}$ & $2.10 \mathrm{D}$ & $2.10 \mathrm{~d}$ & $2.10 \mathrm{~d}$ & $2.30 \mathrm{c}$ & $2.17 \mathrm{D}$ \\
\hline Ca-lactate, $0.5 \%$ & $2.10 \mathrm{de}$ & $2.27 \mathrm{c}$ & $2.33 \mathrm{bc}$ & $2.23 \mathrm{C}$ & $2.30 \mathrm{c}$ & $2.30 \mathrm{c}$ & $2.30 \mathrm{c}$ & $2.30 \mathrm{C}$ \\
\hline Ca-lactate, $1 \%$ & $2.37 \mathrm{~b}$ & $2.50 \mathrm{a}$ & $2.50 \mathrm{a}$ & $2.46 \mathrm{~B}$ & $2.30 \mathrm{c}$ & $2.37 \mathrm{bc}$ & $2.47 \mathrm{ab}$ & $2.38 \mathrm{~B}$ \\
\hline Salicylic acid, $1 \mathrm{mM}$ & $1.10 \mathrm{i}$ & $1.30 \mathrm{~h}$ & $1.57 \mathrm{~g}$ & $1.32 \mathrm{~F}$ & $1.30 \mathrm{~h}$ & $1.30 \mathrm{~h}$ & $1.60 \mathrm{f}$ & $1.40 \mathrm{~F}$ \\
\hline Salicylic acid, $2 \mathrm{mM}$ & $1.00 \mathrm{j}$ & $1.00 \mathrm{j}$ & $1.00 \mathrm{j}$ & $1.00 \mathrm{G}$ & $1.10 \mathrm{i}$ & $1.30 \mathrm{~h}$ & $1.50 \mathrm{fg}$ & $1.30 \mathrm{G}$ \\
\hline Mean & $1.80 \mathrm{C}$ & $1.91 \mathrm{~B}$ & $2.06 \mathrm{~A}$ & & $1.80 \mathrm{c}$ & $1.91 \mathrm{~b}$ & $2.06 \mathrm{a}$ & \\
\hline
\end{tabular}

Note:Differentlettersindicatesiginificantydifferentvalues by ANOVAfollowedbyDuncan testat $\mathrm{P} \leq 0.05$ (small letters refer tovalues recordedineach season, different capitalletters refer tomean values)

Table 9. Microbial count of bacteria $\left(\log \mathrm{CFU}+\mathrm{lg}^{-1}\right)$ of minimally-processed pomegranate arils, $\mathrm{cr}$ Wonderful, as affected by $\mathrm{CaCl}_{2}$ Ca-lactate and salicylic acid treatments during storage at $5 \pm 1^{\circ} \mathrm{C}$ (2013 and 2014seasons)

\begin{tabular}{|c|c|c|c|c|c|c|c|c|}
\hline \multirow{3}{*}{ Treatment } & \multicolumn{4}{|c|}{ Season 2013} & \multicolumn{4}{|c|}{ Season 2014} \\
\hline & \multicolumn{4}{|c|}{ Storage period (days) } & \multicolumn{4}{|c|}{ Storage period (days) } \\
\hline & 0 & 6 & 12 & Mean & 0 & 6 & 12 & Mean \\
\hline Control & $2.77 \mathrm{bc}$ & $2.90 \mathrm{~b}$ & $3.50 \mathrm{a}$ & $3.06 \mathrm{~A}$ & $2.70 \mathrm{bcd}$ & $2.70 \mathrm{bcd}$ & $3.50 \mathrm{a}$ & $2.97 \mathrm{~A}$ \\
\hline $\mathrm{CaCl}_{2}, 0.5 \%$ & $2.67 \mathrm{~cd}$ & $2.60 \mathrm{de}$ & $2.47 \mathrm{e}$ & $2.58 \mathrm{C}$ & $2.67 \mathrm{~cd}$ & $2.00 \mathrm{f}$ & $2.70 \mathrm{bcd}$ & $2.46 \mathrm{C}$ \\
\hline $\mathrm{CaCl}_{2}, 1 \%$ & $2.30 \mathrm{f}$ & $2.70 \mathrm{~cd}$ & $2.77 b c$ & $2.59 \mathrm{C}$ & $2.60 \mathrm{~d}$ & $2.63 \mathrm{~d}$ & $2.67 \mathrm{~cd}$ & $2.63 \mathrm{~B}$ \\
\hline Ca-lactate, $0.5 \%$ & $2.30 \mathrm{f}$ & $2.30 \mathrm{f}$ & $2.73 \mathrm{~cd}$ & $2.44 \mathrm{D}$ & $2.00 \mathrm{f}$ & $2.30 \mathrm{e}$ & $2.83 \mathrm{~b}$ & $2.38 \mathrm{C}$ \\
\hline Ca-lactate, $1 \%$ & $2.70 \mathrm{~cd}$ & $2.73 \mathrm{~cd}$ & $2.70 \mathrm{~cd}$ & $2.71 \mathrm{~B}$ & $2.30 \mathrm{e}$ & $2.80 \mathrm{bc}$ & $2.70 \mathrm{bcd}$ & $2.60 \mathrm{~B}$ \\
\hline Salicylic acid, $1 \mathrm{mM}$ & $2.00 \mathrm{~g}$ & $2.00 \mathrm{~g}$ & $2.00 \mathrm{~g}$ & $2.00 \mathrm{~F}$ & $2.00 \mathrm{f}$ & $2.00 \mathrm{f}$ & $2.30 \mathrm{e}$ & $2.13 \mathrm{D}$ \\
\hline Salicylic acid, $2 \mathrm{mM}$ & $2.00 \mathrm{~g}$ & $2.00 \mathrm{~g}$ & $2.77 \mathrm{bc}$ & $2.26 \mathrm{E}$ & $2.00 \mathrm{f}$ & $2.00 \mathrm{f}$ & $2.63 \mathrm{~d}$ & $2.21 \mathrm{D}$ \\
\hline Mean & $2.39 \mathrm{~B}$ & $2.46 \mathrm{~B}$ & $2.71 \mathrm{~A}$ & & $2.32 \mathrm{~B}$ & $2.36 \mathrm{~B}$ & $2.76 \mathrm{~A}$ & \\
\hline
\end{tabular}

Note:Differentlettersindicatesignificantlydifferent values by ANOVA followed by Duncan testat $\mathrm{P} \leq 0.05$ (small letters refer tovalues recorded in each season, different capital letters refer to mean values)

Table 10. Sensory evaluation (score) of minimally-processed pomegranate arils, cv Wonderful, as affected by CaCl2, Ca-lactate and salicylic acid treatments after 12 days of storage at $5 \pm 1^{\circ} \mathrm{C}$ (2013 and 2014 seasons). GA, general appearance

\begin{tabular}{|c|c|c|c|c|c|c|c|c|}
\hline \multirow{2}{*}{ Treatment } & \multicolumn{4}{|c|}{ Season 2013} & \multicolumn{4}{|c|}{ Season 2014} \\
\hline & GA & Decay & Flavor & Off odors & GA & Decay & Flavor & Off odors \\
\hline Control & $7.17 \mathrm{~b}$ & $2.00 \mathrm{a}$ & $3.75 \mathrm{~b}$ & $1.58 \mathrm{a}$ & $6.83 c$ & $2.00 \mathrm{a}$ & $3.58 \mathrm{~b}$ & $2.00 \mathrm{a}$ \\
\hline $\mathrm{CaCl} 2,0.5 \%$ & $7.83 \mathrm{ab}$ & $1.00 \mathrm{~b}$ & $4.33 \mathrm{a}$ & $1.00 \mathrm{~b}$ & $7.67 \mathrm{abc}$ & $1.00 \mathrm{~b}$ & $4.33 \mathrm{a}$ & $1.00 \mathrm{~b}$ \\
\hline $\mathrm{CaCl} 2,1 \%$ & $7.67 \mathrm{ab}$ & $1.00 \mathrm{~b}$ & $4.25 \mathrm{a}$ & $1.00 \mathrm{~b}$ & $7.67 \mathrm{abc}$ & $1.00 \mathrm{~b}$ & $4.25 \mathrm{a}$ & $1.00 \mathrm{~b}$ \\
\hline Ca-lactate, $0.5 \%$ & $7.67 \mathrm{ab}$ & $1.08 \mathrm{~b}$ & $3.92 \mathrm{ab}$ & $1.00 \mathrm{~b}$ & $7.67 \mathrm{abc}$ & $1.17 \mathrm{~b}$ & $3.97 \mathrm{ab}$ & $1.67 \mathrm{~b}$ \\
\hline Ca-lactate, $1 \%$ & $7.67 \mathrm{ab}$ & $1.00 \mathrm{~b}$ & $4.00 \mathrm{ab}$ & $1.00 \mathrm{~b}$ & $7.67 \mathrm{abc}$ & $1.08 \mathrm{~b}$ & $4.00 \mathrm{ab}$ & $1.08 \mathrm{~b}$ \\
\hline Salicylic acid, $1 \mathrm{mM}$ & $8.17 \mathrm{a}$ & $1.00 \mathrm{~b}$ & $4.25 \mathrm{a}$ & $1.00 \mathrm{~b}$ & $8.30 \mathrm{a}$ & $1.08 \mathrm{~b}$ & $4.25 \mathrm{a}$ & $1.00 \mathrm{~b}$ \\
\hline Salicylic acid, $2 \mathrm{mM}$ & $8.00 \mathrm{a}$ & $1.00 \mathrm{~b}$ & $4.25 \mathrm{a}$ & $1.00 \mathrm{~b}$ & $8.00 \mathrm{a}$ & $1.00 \mathrm{~b}$ & $4.25 \mathrm{a}$ & $1.00 \mathrm{~b}$ \\
\hline
\end{tabular}

Note: different letters indicate significantly different values by ANOVA followed by Duncan test at $\mathrm{P} \leq 0.05$ 
514

\section{Colormeasurements}

$\mathrm{L}^{*}$ value

With regard to color characteristics of pomegranate arils, the $\mathrm{L}^{*}$ value showed a significant decrease along storage period up to 9 days in both studied seasons (Table 6), indicating a darker coloration, in accordance with previous reports (Toor and Savage, 2006; Ashebir et al., 2009; Sanchís et al., 2015). As for the different treatments, no significant difference was observed. In accordance, Khademi et al. (2012) reported that SA at 1 and $2 \mathrm{mM}$ determined no significant difference of color index among treatments, and also Sepúlveda et al. (2000) and Artés et al. (2000) observed no color change in minimally processed 'Wonderful' arils stored at $4 \pm 0.5^{\circ} \mathrm{C}$ in semi- permeable films for 14 days. Gil et al. (1996) reported a relatively small change in $\mathrm{L}^{*}$ values for 'Mollar' arils, packed in oriented polypropylene (OPP) bags stored at 8, 4 and $1{ }^{\circ} \mathrm{C}$ for 7 days. These results are also in agreement with the pattern of decline in anthocyanins, observed by Meighani et al. (2014).

\section{Hue angle $\left(h^{\circ}\right)$}

As for of hue angle $\left(h^{\circ}\right)$, Table 7 shows that values regularly decreased along the storage period. In both seasons, at the end of the storage period the $h^{\circ}$ values were significantly lower than at time ' 0 '. No significant difference in $h^{\circ}$ mean values was observed among the different treatments and untreated arils, similarly with what previously reported by Mirdehghan et al. (2012) and Artés et al. (2000). Also Belay et al. (2016) didn't obtain significant differences between treatments with atmospheric oxygen at the end of 12 days of cold storage $\left(5^{\circ} \mathrm{C}\right)$ of minimally processed pomegranate arils.

\section{Microbialquality}

Total fungal and bacterial counts were assessed at the beginning of the trial (day 0 ), then at 6 and 12 days of storage. In both seasons, Tables 8 and 9 show a slight increment, but often significant, in both fungal and bacterial counts during the storage period. However, counts remained fewer than $5 \log \mathrm{CFU} \mathrm{g}{ }^{-1}$, which is the maximum limit for yeasts and moulds in raw and fresh-cut fruits by the South African legislation (FCD, Act 54 1979; Caleb et al., 2013). Data also show that control arils had always the highest count of fungi/yeasts and bacteria, with significant mean values. Lowest counts were obtained with SA treatment at both the tested concentrations, with significant differences with the other treatments and untreated arils. Among the different treatments, $1 \%$ Ca-lactate was the one that produced the highest counts of fungi/yeasts and bacteria both along the storage period, and as mean values.

Overall, data obtained in these trials were in agreement with those reported by Arendse et al. (2014). Several types of moulds and bacteria are associated with pomegranate fruit, affecting its overall quality. In addition, Soliva-Fortuny and Martín-Belloso (2003) reported that physicochemical properties of fruit, such as $\mathrm{pH}$ and TA, have an important effect on microbial shelf-life of fresh-cut fruit.

\section{Sensory evaluation}

Sensory descriptive analysis, coupled with a consumer preference test, can establish the relative importance of the characteristics that drive acceptability. Difference tests can be used to select the best treatments for use in a consumer preference test (Barrett et al., 2010). As shown in Table 10, data indicate that arils treated with SA ( 1 and $2 \mathrm{mM}$ ) maintained the best general appearance up to the end of the storage period, as the scores were significantly higher in comparison with untreated ones. However, differences were not significant among the various treatments. In both seasons, all treatments showed a minimal or nil visible decay after 12 days of storage, while control arils recorded a slight visible decay.

Flavor may be evaluated with either instrumental or sensory methods, but most scientists agree that sensory methods are the most critical for this particular quality attribute (Barrett et al., 2010). Both calcium (0.5\% and 1\%) and SA (1 and $2 \mathrm{mM}$ ) treatments obtained the highest scores of flavors, while untreated (control) arils showed the lowest score, with no significant differences with arils treated with Ca-lactate at both tested concentrations.

Untreated arils (control) recorded the highest scores of off odor detection. On the other hand, differences between treatments failed to show any significance, although $0.5 \% \mathrm{Ca}$ lactate showed a slight increase in the off odour in the second season of investigation.

\section{Conclusions}

Treatments with different compounds can help the maintenance of post-harvest quality of fruits. However, in order to achieve maximum time of conservation, the best combination compound/concentration should be always tested. In this study, the post-harvest assessment was performed at 3-day intervals with several parameters (appearance, decay, off odor, flavor, total soluble solids, acidity, anthocyanin and vitamin C content, firmness, color development by $L^{*}$ value and hue angle), as well as with microbial load assessed at 0,6 and 12 days of storage, achieving a clear view of the effectiveness of calcium chloride, $\mathrm{Ca}$ lactate and SA in the maintenance of pomegranate aril quality. Based on the data it can be concluded that SA had a significant effect on quality parameters changes and sensorial quality of pomegranate arils, determining the lowest microbial counts and the highest values in all the tested quality attributes.

\section{References}

Aarabi A, Barzegar M, Azizi MH (2008). Effect of cultivar and cold storage of pomegranate (Punica granatum L.) juices on organic acid composition. ASEAN Food Journal 15:45-55.

Agar IT, Hess-Pierce B, Kader AA (1999). Postharvest $\mathrm{CO}_{2}$, ethylene production and quality maintenance of fresh-cut kiwifruit slices. Journal of Food Science 64(3):433-440.

Aguayo E, Escalona VH, Artés F (2008). Effect of hot water treatment and various calcium salts on quality of fresh-cut 'Amarillo' melon. Postharvest Biology and Technology 47:397-406.

Aguayo E, Jansasithorn R, Kader A (2012).Effects of calcium chloride dip and 1-methylcyclopropene on quality changes in arils from stored pomegranates. In: Melgarejo P, Valero D (Eds).II International Symposium on the Pomegranate. Zaragoza. CIHEAM/Universidad Miguel Hernándezpp 195-199.

Aguayo E, Requejo C, Stanley R, Woolf A (2010). Effects of calcium ascorbate treatments and storage atmosphere on antioxidant activity and quality of fresh-cut apple slices. Postharvest Biology and Technology 57:52-60. 
Amith PK, Mini C, Manju RV (2015). Protocol development for minimally processed pomegranate arils. International Journal Proceedings and Postharvest Technology 6(1):62-68.

A.O.A.C. (1990). Official Methods of Analysis. 15th Ed. Method 967(17):923.

Arendse E, Fawole OA, Opara UL (2014). Effects of postharvest storage conditions on phytochemical and radical-scavenging activity of pomegranate fruit (cv. Wonderful). Scientia Horticulturae 169:125129.

Arhtar A, Abbasi NA, Hussain A (2010). Effect of calcium chloride treatments on quality characteristics of loquat fruit during storage. Pakistan Journal of Botany 42:181-188.

Artés F, Villaescusa R, TudelaJA (2000). Modified atmosphere packaging of pomegranates. Journal of Food Science 65:1112-1116.

Asghari M, Aghdam M (2010). Impact of salicylic acid on postharvest physiology of horticultural crops. Trends in Food Science and Technology 21:502-509.

Ashebir D, Jezik K, Weingartemann H, Gretzmacher R (2009). Change in color and other fruit quality characteristics of tomato cultivars after hotair drying at low final-moisture content. International Journal of Food Sciences and Nutrition 60:308-315.

Babalar M, Asghari M, Talaei A, Khosroshahi A (2007). Effect of pre- and postharvest salicylic acid treatment on ethylene production, fungal decay and overall quality of Selva strawberry fruit. Food Chemistry 105:449453.

Barrett DM, Beaulieu JC, Shewfelt R (2010). Color, flavor, texture, and nutritional quality of fresh-cut fruits and vegetables: desirable levels, instrumental and sensory measurement, and the effects of processing. Critical Reviews in Food Science and Nutrition 50(5):369-389.

Beaulieu JC, Gorny JR (2002). Fresh-cut fruits. In: Gross KC, Wang CY, Saltveit ME (Eds). The Commercial Storage of Fruits, Vegetables, Florist and Nursery Stocks. Handbook 66. USDA, Washington, D.C.

Beckers GJM, Spoel SH (2006). Fine-tuning plant defence signalling: salicylateversus jasmonate. Plant Biology 8:1-10.

Belay ZA, Caleb OJ, Opara UL (2016). Impacts of low and superatmospheric oxygen concentrations on quality attributes, phytonutrient content and volatile compounds of minimally processed pomegranate arils (cv. Wonderful). Postharvest Biology and Technology 124:119127.

Biten Court De Souza AL, Quintao Scalon SDP, Chitarra MIF, Chitarra $\mathrm{AB}$ (1999). Postharvest application of $\mathrm{CaCl}_{2}$ in strawberry fruits (Fragaria ananassa Dutch cv. Sequoia): evaluation of fruit quality and postharvest life. Ciência Agrotecnologia 23:841-848.

Caleb OJ, Opara UL, Pramod VM, Manley M, Rena M, Mokwena L, Tredouxe AGJ (2013). Effect of modified atmosphere packaging and storage temperature on volatile composition and postharvest life of minimally-processed pomegranate arils (cvs. 'Acco' and 'Herskawitz'). Postharvest Biology and Technology 79:54-61.

CalebOJ, Opara UL, Witthuhn C (2012). Modified atmosphere packaging of pomegranate fruit and arils: a review. Food and Bioprocess Technology 5(1):15-30.

Coultate TP (2007). Food: The Chemistry of Its Components. RSC Publishing, UK.
Demarty M, Morvan C, Thellier M (1984). Ca and the cell wall. Plant, Cell and Environment 7:441-447.

Ding ZS, Tian SP, Zheng XI, Zhou, ZW, Xu Y (2007). Responses of reactive oxygen metabolism and quality in mango fruit to exogenous oxalic acid or salicylic acid under chilling temperature stress. Physiologia Plantarum 130:112-121.

Dong X, Wrolstad RE, Sugar D (2000). Extending shelf-life of fresh-cut pears. Journal of Food Science 65:181-186.

Dowson JW (1957). Plant disease due to bacteria. 2nd edition. Cambridge University Press, London.

El-Bassiouny REI (2003). Modified atmosphere polyethylene packages maintain the quality of snap bean pods during storage. Journal of Agricultural Science 28(5):3550-3571.

El-Kassas SE, Mahmoud HM, Salhy AM, Mohamed SA (1995). Effect of $\mathrm{GA}_{3}$ and calcium treatments on the keeping quality and storability of 'Manfalouty' pomegranate fruits. Assiut Journal of Agricultural Science 26:4.

Elyatem SM, Kader AA (1984). Postharvest physiology and storage behavior of pomegranate fruits. Scientia Horticulturae 24:287-298.

Fawole OA, Opara UL (2013). Effects of storage temperature and duration on physiological responses of pomegranate fruit. Industrial Crops and Products 47:300-309.

Ferguson IB (1984). Calcium in plant senescence and fruit ripening. Plant Cell \&Environment 7:477-489.

Gaffney T, Friedrich, L, Vernooij B, Negrotto D, Nye G, Uknes S, Ward E, Kessmann H, Ryals J (1993). Requirement of salicylic acid for the induction of systemic acquired resistance. Science 261:754756.

Garcia JM, Herrea S, Morilla A (1996). Effects of postharvest dips in calcium chloride on strawberry. Journal of Agricultural and Food Chemistry 44:30-33.

García-Viguera C,Zafrilla P, ArtésF, RomeroF, Abellán P, Tomás-Barberán FA (1998). Colour and anthocyanin stability of red raspberry jam. Journal of the Science of Food and Agriculture 78:565-573.

Gil MI, Martínez JA, Artés F (1996). Minimally processed pomegranate seeds. LWT - Food Science and Technology 29:708-713.

Gorny JR, Gil MI, Kader AA (1998). Postharvest physiology and quality maintenance offresh cut pears. Acta Horticulturae 464:231-236.

Gorny JR, Hess-Pierce B, Cifuentes RA, Kader AA (2002). Quality changes in fresh-cut pear slices as affected by controlled atmospheres and. chemical preservatives. Postharvest Biology Technology 24(3):271-278.

Gorny JR, Hess-Pierce B, Kader AA (1999). Quality changes in fresh-cut peach and nectarine slices as affected by cultivar, storage atmosphere and chemical treatments. Journal of Food Science 64:429-432.

Holland D, Hatib K, Bar-Yảakov I (2009). Pomegranate: botany, horticulture, breeding. In: Janick J (Ed). Horticultural Reviews, vol. 35. John Wiley \& Sons Inc., USA pp 127-191.

Hooper L, Cassidy A (2006). A review of the health care potential of bioactive compounds. Journal of the Science Food and Agriculture 86:1805-1813.

Hsia CL, Luh BS, Chichester CO (1965). Anthocyanins in freestone peaches.Journal of Food Science 30:5-12.

Huang RH, Liu JH, Lu Y, Xia RX (2008). Effect of salicylic acid on the antioxidant system in the pulp of 'Cara cara' navel orange (Citrus sinensis 
516

L. Osbeck) at different storage temperatures. Postharvest Biology and Technology 47:168-175.

Hussein MAH, Hussein MAS (1972). Suitability of pomegranate varieties for processing. Assiut Journal of Agricultural Science 3:303-307.

Johnson LF, Curl RA, Bond JH, Fribourg HA (1960). Methods for studying soil microflora - plant disease relationships. 2nd edition, Minneapolis, Burgess publishingCo.

Kader AA, Lipton WJ, Morris LL (1973). System for scoring quality of harvested lettuce. Horticultural Science (8):408-409.

Kazemi M, Aran M, Zamani S (2011). Effect of calcium chloride and salicylic acid treatments on quality characteristics of kiwifruit (Actinidia deliciosa, cv. Hayward) during storage. American Journal of Plant Physiology 6:183-189.

Khademi O, Zamani Z, Mostofi Y, Kalantari S, Ahmadi A (2012). Extending storability of persimmon fruit, cv. Karaj, by postharvest application of salicylic acid. Journal of Agricultural Science and Technology 14:1067-1074.

Khademi Z, Ershadi A (2013). Postharvest application of salicylic acid improves storability of peach (Prunus persica cv. Elberta) fruits. International Journal of Agriculture and Crop Sciences 5(6):651-655.

Kirkby EA, Pilbeaam DJ (1984). Calcium as a plant nutrient. Plant, Cell \& Environment 7:397-405.

Küpper W (1995). Wirkungen von Temperatur und $\mathrm{CO}_{2}$-Konzentration in der Langfristigen CA-Lagerung auf Verschiedene Qualitaetsmerkmale und die Respiration Waehrend der Nachlagerungsphase des Granatapfels (Punica granatum L.) der Sorte 'Hicaznar.' PhD Thesis. Institut für Obstbau und Gemüsebau der Rheinschen, Friedrich-Wilhelms-Univertat, Bonn, Germany.

Lara I, García P, Vendrell M (2004). Modifications in cell wall composition after cold storage of calcium-treated strawberry (Fragaria $\times$ ananassa Duch.) fruit. Postharvest Biology and Technology 34:331-339.

Li Z, Pan Q, Cui X, Duan C (2010). Optimization on anthocyanins extraction from wine grape skins using orthogonal test design. Food Science and Biotechnology 19(4):1047-1053.

Lucas GH (1944). Determining ascorbic acid in large number of plant samples. Industrial and Engineering Chemistry Research 16:649-652.

Luna-Guzman I, Barrett DM (2000). Comparison of calcium chloride and calcium lactate effectiveness in maintaining shelf stability and quality of fresh-cut cantaloupes. Postharvest Biology and Technology 19:61-72.

Mahajan BVC, Dhatt AS (2004). Studies on postharvest calcium chloride application on storage behaviour and quality of Asian pear during cold storage. Journal of Food, Agriculture and Environment 2:157-159.

Malamy J, CarrJP, KlessigDF, Raskin I (1990). Salicylic acid: a likely signal in the resistance response of tobacco to viral infection. Science 250:10021004.

Manganaris GA, Vasilakakis M, Diamantidis G, Mignani I (2007). The effect of postharvest calcium application on tissue calcium concentration quality attributes incidence of flesh browning and cell wall physicochemical aspects of peach fruits. Food Chemistry 100:13851392.

Massantini R, Kader AA (1995).Storability and quality preservation of sliced kiwifruits (Conservazione e mantenimento qualitativo delle fette di kiwi; in Italian).Industrie Alimentari34:357-360.
MedinaMS, Tudela JA, Marín A, Allende, Gil MI (2012). Short postharvest storage under low relative humidity improves quality and shelf life of minimal processed baby spinach. Postharvest Biology and Technology 67:1-9.

Meighani H, Ghasemnezhad M, Bakhshi D (2014). Evaluation of biochemical composition and enzyme activities in browned arils of pomegranate fruits. International Journal of Horticultural Science and Technology 1(1):53-65.

Mirdehghan SH, Ghotbi F (2014). Effects of salicylic acid, jasmonic acid and calcium chloride on reducing chilling injury of pomegranate (Punica granatum L.). Journal of Agricultural Science and Technology 16:163173.

Mirdehghan SH, Vatanparast G, Karim HR, Vazifeshenas MH (2012). Preharvest foliar application of methyl jasmonate, salicylic acid and potassium sulfate on improving the quality of pomegranate fruit. Options Méditerranéennes 103:183-189.

Mphahlele RR, Fawole OA, Stander MA, Opara UL (2014). Preharvest and postharvest factors influencing bioactive compounds in pomegranate (Punica granatum L.): a review. Scientia Horticulturae 178:114123.

O'Grady L, Sigge G, Caleb OJ, Opara UL (2014). Effects of storage temperature and duration on chemical properties, proximate composition and selected bioactive components of pomegranate (Punica granatum L.) arils. LWT - Food Science and Technology 57:508-515.

Opara UL, Al-Ani MR, Al-Shuaibi YS (2009). Physicochemical properties, vitamin $\mathrm{C}$ content, and antimicrobial properties of pomegranate fruit (PunicagranatumL.). Food Bioprocess Technology 2:315-321.

Picchioni GA, Watada AE, Conway WS, Whitaker B, Sams CE (1998). Postharvest calcium infiltration delays membrane lipid catabolism in apple fruit.Journal of Agricultural and Food Chemistry 46:2452-2457.

PontingJD, Jackson R, Watters G (1971). Refrigerated apple slices: effects of $\mathrm{pH}$, sulfites and calcium on texture. Journal of Food Science 36:449450.

Ponting JD, Jackson R, Watters G (1972). Refrigerated apple slices: preservative effects of ascorbic acid, calcium and sulfites. Journal of Food Science 37:434-435.

Poovaiah BW (1986). Role of calcium in prolonging the storage-life of fruits and vegetables. Food Technology 40:86-89.

Ranjbaran E, Sarikhani H, Bakhshi D, Mehrdad P (2011). Investigation of salicylic acid application to reduce postharvest losses in stored 'Bidaneh Ghermez' table grapes. International Journal of Fruit Science 11:430439.

Raskin I (1992). Salicylate, a new plant hormone. Plant Physiology 99:799803.

Sanchís E, Milagros M, María B, Pérez-Gago (2015). Effect of maturity stage at processing and antioxidant treatments on the physico chemical, sensory and nutritional quality of fresh-cut 'Rojo Brillante' persimmon. Postharvest Biology and Technology 105:3444.

Sayyari M, Babalar M, Kalantari S, Serrano M, Valero D (2009). Effect of salicylic acid treatment on reducing chilling injury in stored pomegranates. Postharvest Biology and Technology 53:152-154.

Sayyari M, Salvador C, Daniel V, Huertas MD, Maria S (2011). Acetyl 
salicylic acid alleviates chilling injury and maintains nutritive and bioactive compounds and antioxidant activity during postharvest storage of pomegranates. Postharvest Biology and Technology 60:136142.

Sepúlveda E, Galletti L, Sáenz C, Tapia M (2000). Minimal processing of pomegranate var. Wonderful. Options Mediterraneenes 42:237-242.

Shafiee M, Taghavi TS, Babalar M (2010). Addition of salicylic acid to nutrient solution combined with postharvest treatments (hot water, salicylic acid, and calcium dipping) improved postharvest fruit quality of strawberry. Scientia Horticulturae 124:40-45.

Snedecor GW, Cochran WG (1982). Statistical Methods. $7^{\text {th }}$ Ed. The Iowa State Univ.Press, Ames Iowa, USA.

Sogvar OB, Saba MK, Emamifar A (2016). Aloe vera and ascorbic acid coatings maintain postharvest quality and reduce microbial load of strawberry fruit. Postharvest Biology and Technology 114:29-35.

Soliva-Fortuny RC, Martín-Belloso O (2003). New advances in extending shelf-life of fresh-cut fruits: a review. Trends in Food Science and Technology 14:341-353.

Srivastava MK, Dwivedi UN (2000). Delayed ripening of banana fruit by salicylic acid. Plant Science 158:87-96.

Terry LA, Joyce DC (2004). Elicitors of induced disease resistance in postharvest horticulture crops: a brief review. Postharvest Biology and Technology 32:1-13.

Toor RK, Savage GP (2006). Effect of semi-drying on the antioxidant components of tomatoes. Food Chemistry 94:90-97.
Veltman RH, Kho RM, Van Schaik ACR, Sanders MG, Oosterhaven J (2000). Ascorbic acid and tissue browning in pears (Pyrus communis $L$. cvs Rocha and Conference) under controlled atmosphere conditions. Postharvest Biology and Technology 19:129-137.

Viuda-Martos M, Fernández-López J, Pérez-Álvarez JA (2010). Pomegranate and its many functional components as related to human health: a review. Comprehensive Reviews in Food Science and Food Safety 9:635-665.

Wang L, Chen S, Kong W, Li S, Archbold DD (2006). Salicylic acid pretreatment alleviates chilling injury and affects the antioxidant system and heat shock proteins of peaches during cold storage. Postharvest Biology and Technology 41:244251.

White PJ, Broadley MR (2003). Calcium in plants. Annals of Botany 92:487-511.

Withy LM, Nguyen TT, Wrolstad RE, Heatherbell DA (1993). Storage changes in anthocyanin content of red raspberry juice concentrate. Journal of Food Science 58:190-192.

Xu X, Tian S (2008). Salicylic acid alleviated pathogen-induced oxidative stress in harvested sweet cherry fruit. Postharvest Biology and Technology 49:379-385.

Yao H, Tian S (2005). Effects of pre- and postharvest application of salicylic acid or methyl jasmonate on inducing disease resistance of sweet cherry fruit in storage. Postharvest Biology and Technology 35:253-262.

Zhang Y, Chen K, Zhang S, Ferguson I (2003). The role of salicylic acid in postharvest ripening of kiwifruit. Postharvest Biology and Technology 28:67-74. 